\title{
Wideband Channel Estimation and Prediction in Single-Carrier Wireless Systems
}

\author{
Wei Liu, Lie-Liang Yang and Lajos Hanzo \\ School of ECS, University of Southampton, SO17 1BJ, UK. \\ Tel: +44-23-8059 3125, Fax: +44-23-8059 4508 \\ Email: w103r, lly, 1h@ecs.soton.ac.uk; http://www-mobile.ecs.soton.ac.uk
}

\begin{abstract}
In this contribution wideband channel estimation and prediction designed for single-carrier wideband wireless communications systems are investigated. Specifically, the single-carrier wideband pilot signal received by the receiver is first converted to the frequency-domain. Then, the envelope of the channel transfer function (CTF) is estimated in the frequency-domain, in order to reduce the effects of background noise on the channel prediction step to be invoked. Finally, channel prediction is carried out based on the estimated CTF in the frequency-domain, where a Kalman filter assisted long-range channel prediction algorithm is employed. Our simulation results show that for a reasonable signal-to-noise ratio (SNR) value the proposed frequency-domain based wideband channel estimator is capable of efficiently mitigating the effects of the background noise, hence enhancing the performance of wideband channel prediction.
\end{abstract}

\section{INTRODUCTION}

Adaptive modulation is capable of substantially improving the achievable system performance, provided that the relevant channel state information (CSI) can be accuratelly predicted [1], [2]. In future wireless systems, the carrier frequency is likely to be high, which results in a high Doppler frequency. For systems using CSI feedback, the outdated information estimated based on the past data may not be sufficiently accurate. In this case we may have to know the CSI in advance, which can be achieved with the aid of long range CSI prediction [3].

In the context of wideband channel prediction [4], [5], [6], amongst others subspace based algorithms have been investigated. In [4] the ESPRIT algorithm has been employed for the prediction of fast fading wideband channels. Since the time-domain variations of the wideband channel transfer functions (CTF) are typically highly correlated, the CTF values encountered at different frequencies may be recorded during an observation segment and used in the ESPRIT algorithm for wideband channel prediction. More specifically, it was shown in [4] that the complex path gains may be determined by solving a set of linear equations. Finally, the CTF can be extrapolated in order to predict the future CTF. In [5], [6] both the one-dimentional 1-D and 2-D Unitary-Esprit algorithms have been employed for estimating the CTF. Once the CTF has been determined, its future values can be extrapolated in both the time and frequency domain.

An attractive technique designed for the estimation or prediction of wideband channels is based on transfering the received signal from the time-domain to the frequency-domain and estimating or predicting it in the frequency domain [2]. More specifically, in the frequency-domain the available frequency band can be divided into a number of subbands, each having a bandwidth lower than the coherence bandwidth of the channel, so that signals transmitted in each subband experience flat fading. In this case, each of the subchannels can be estimated or predicted using a variety of schemes designed for flat fading channels. Specifically, each subchannel can be predicted using for example the

The financial support of the EPSRC, UK and that of the EU under the auspices of the Phoenix and Newcom projects is gratefully acknowledged. long-range prediction techniques of [3] proposed for narrowband fading channels.

In this contribution we investigate both wideband channel estimation and prediction designed for single-carrier communications systems. Specifically, in our scheme the received pilot signal is first transformed to the frequency-domain, where the CTF of the wideband channel is estimated. Then, the future CTFs of the wideband channel are predicted with the aid of the current as well as previous CTFs estimated in the frequency-domain (FD), using a Kalman filter based long-range channel prediction scheme.

The outline of the paper is as follows. Section II briefly characterises the family of wideband channels, while Section III describes our transmission scheme. The topic of Section IV is channel estimation and prediction, while our results are discussed in Section V

\section{WidEBAND WIRELESS CHANNELS}

In wideband wireless communications the received signal can be expressed as

$$
r(t)=\sum_{k=-\infty}^{+\infty} b_{k} c\left(t ; t-k T_{b}\right)+z(t)
$$

where $b_{k}$ represents the discrete-time transmitted signal, $T_{b}$ is the symbol period and $z(t)$ is the AWGN component having zero mean and a variance of $N_{0} / 2$. In (1) $c(t ; \tau)$ represents the combined channel impulse response (CIR), which can be expressed as

$$
c(t ; \tau)=g(t) \otimes g(t ; \tau) \otimes g^{*}(-t),
$$

where $g(t)$ and $g^{*}(-t)$ represent the pulse shaping filter at the transmitter and the corresponding matched filter at the receiver, respectively, while $g(t ; \tau)$ represents the physical time-varying frequencyselective fading channel's impulse response (CIR).

Furthermore, in (2) the operator $\otimes$ denotes the convolution operation. When $r(t)$ of (1) is sampled at the symbol rate, we obtain a discrete-time received signal, which can be expressed as

$$
\left.r_{n} \equiv r(t)\right|_{t=n T_{b}}=\sum_{k=-\infty}^{+\infty} c_{(n ; k)} b_{(n-k)}+z_{n} .
$$

Equation (3) suggests that the combined impulse response $c_{(n ; k)}$ may extend to infinity. However, in communications applications it is general practice to truncate it to a finite duration $L$, yielding the discretetime model represented by

$$
r_{n}=\sum_{k=0}^{L-1} c_{(n ; k)} b_{(n-k)}+z_{n} .
$$




\section{Single CARrier DATA TRANSMission}

In single carrier block-based data transmission over multipath fading channels, there are $N$ useful data symbols, namely $\left[b_{(k ; 0)}, b_{(k ; 1)}, \cdots, b_{(k ; N-1)}\right]$, in the $k$ th transmission block. However, in order to eliminate the dispersion-induced interblock interference (IBI), often a cyclic prefix consisting of $u \geq L-1$ symbols [7] is appended to these $N$ useful data symbols. For the sake of aiding channel prediction, the $u$-symbol cyclic prefix is often chosen to be the same as the last $u$ useful data symbols in each length- $N$ transmission block. Consequently, a transmission block is comprised of $(N+u)$ data symbols, namely of $\left[b_{(k ; N-u)}, \cdots, b_{(k ; N-1)}, b_{(k ; 0)}, b_{(k ; 2)}, \cdots, b_{(k ; N-1)}\right]$. Let $T_{B}$ be the duration of a transmission block, and $T_{b}$ be the duration of a data symbol, then we have

$$
T_{B}=(N+u) T_{b} .
$$

We assume that the CIR encountered is time-invariant during the transmission of a transmission block [7], [8], [9]. Furthermore, we assume that for the $k$ th transmission block the CIR vector $\mathbf{c}_{k}$ may be expressed as

$$
\mathbf{c}_{k}=\left[c_{(k ; 0)}, c_{(k ; 1)}, \cdots, c_{(k ; L-1)}\right]^{T},
$$

where the superscript $T$ represents the transpose, and

$$
c_{(k ; l)}=c\left(k T_{B} ; l T_{b}\right)
$$

denotes the channel's amplitude sampled at the time instant of the $l$ th data symbol within the $k$ th transmission block. According to (4) and (7), the received signal samples of the $k$ th transmission block can be expressed as

$$
r_{(k ; n)}=\sum_{l=0}^{L-1} c_{(k ; l)} b_{(k ; n-l)}+z_{(k ; n)}, n=0, \cdots, N+u-1 .
$$

Having received $(N+u)$ number of samples corresponding to a transmission block, the first $u$ samples are discarded, in order to eliminate the IBI. Consequently, the $N$ received samples corresponding to the $k$ th transmission block after removing the cyclic prefix can be expressed as

$$
\mathbf{r}_{k}=\mathbf{C}_{k} \mathbf{b}_{k}+\mathbf{z}_{k},
$$

where $\mathbf{r}_{k}$ consists of the $N$ number of samples, which is expressed as

$$
\mathbf{r}_{k}=\left[r_{(k ; 0)}, r_{(k ; 1)}, \cdots, r_{(k ; N-1)}\right]^{T},
$$

$\mathbf{z}_{k}$ is an $N$-dimensional noise vector expressed as

$$
\mathbf{z}_{k}=\left[z_{(k ; 0)}, z_{(k ; 1)}, \cdots, z_{(k ; N-1)}\right]^{T}
$$

and $\mathbf{b}_{k}$ is an $N$-dimensional vector containing the $N$ number of transmitted data symbols within the $k$ th transmission block, which is given by

$$
\mathbf{b}_{k}=\left[b_{(k ; 0)}, b_{(k ; 1)}, \cdots, b_{(k ; N-1)}\right]^{T} .
$$

Finally, in (9) $\mathbf{C}_{k}$ is an $(N \times N)$-dimensional circulant matrix with the first column containing the CIR extended by $(N-L)$ zeros [8].

Since $\mathbf{C}_{k}$ is a circulant matrix, it can be shown that $\mathbf{C}_{k}$ may be expressed as [8]

$$
\mathbf{C}_{k}=\mathbf{Q}^{H} \Lambda \mathbf{Q},
$$

where the superscript $H$ represents the conjugate transpose operation, and $\mathbf{Q}$ is the $(N \times N)$-dimensional orthonormal discrete Fourier transform (DFT) matrix satisfying $\mathbf{Q} \mathbf{Q}^{H}=\mathbf{I}$.

In (13) $\Lambda$ is a diagonal matrix, whose $(n, n)$ th element is equal to the $n$th DFT factor $\breve{c}_{(k ; n)}$ of $\left[c_{(k ; 0)}, c_{(k ; 1)}, \cdots, c_{(k ; L-1)}\right]$, which can be expressed as

$$
\breve{c}_{(k ; n)}=\sum_{l=0}^{L-1} c_{(k ; l)} e^{-j 2 \pi \frac{l n}{N}}, \quad 0 \leq n \leq N-1 .
$$

Notice that $\breve{c}_{(k ; n)}$ in (14) is in fact the discrete CTF.

Upon applying the DFT to the received samples $\mathbf{r}_{k}$, we obtain the frequency domain received signal vector, which can be expressed as

$$
\breve{\mathbf{r}}_{k}=\Lambda \breve{\mathbf{b}}_{k}+\breve{\mathbf{z}}_{k}
$$

where $\breve{\mathbf{b}}_{k}=\sqrt{N} \mathbf{Q} \mathbf{b}_{k}$ represents the DFT of the input symbol vector $\mathbf{b}_{k}$ shown in (12), while $\breve{\mathbf{z}}_{k}=\sqrt{N} \mathbf{Q} \mathbf{z}_{k}$ is the DFT of the noise vector $\mathbf{z}_{k}$ of (11). When the noise sample $z_{k}$ is assumed to be generated by an AWGN process having a variance of $N_{0} / 2$, the covariance matrix of $\mathbf{z}_{k}$ is $N_{0} / 2 \mathbf{I}_{N}$. Consequently, the covariance matrix of the discrete time noise process $\breve{\mathbf{z}}_{k}$ can be computed according to $E\left[\breve{\mathbf{z}}_{k} \breve{\mathbf{z}}_{k}^{H}\right]=N N_{0} / 2 \mathbf{I}_{N}$. Hence, the discrete time noise vector $\breve{\mathbf{z}}_{k}$ is still a white Gaussian vector.

\section{Channel Estimation and Channel Prediction}

In practice only noisy channel samples are available and the noise has a detrimental impact on the performance of the prediction. Hence, in order to mitigate the effect of noise contamination on prediction, channel estimation is typically carried out prior to channel prediction.

\section{A. Channel Estimation in the Frequency Domain}

In the context of single carrier systems, when the pilot symbol block is received, the time-domain pilot information can be converted to the frequency-domain by using the DFT, as argued in Section III. According to (15), the frequency domain pilot signal $\breve{r}_{(k ; n)}$ can be expressed as

$$
\breve{r}_{(k ; n)}=\breve{c}_{(k ; n)} \breve{b}_{(k ; n)}+\breve{z}_{(k ; n)} .
$$

Upon dividing both sides of (16) by $\breve{b}_{(k ; n)}, \tilde{c}_{(k ; n)}$, namely of the estimate of $\breve{c}_{(k ; n)}$, can be expressed as

$$
\tilde{c}_{(k ; n)}=\frac{\breve{r}_{(k ; n)}}{\breve{b}_{(k ; n)}}=\breve{c}_{(k ; n)}+\frac{\left.\breve{z}_{(k ; n)}\right)}{\breve{b}_{(k ; n)}}, n=0,1, \cdots, N-1 .
$$

The autocorrelation function of $\breve{c}_{(k ; n)}$ can be expressed as [10]

$$
r[\Delta k ; \Delta n]=E\left[\breve{c}_{(k ; n)} \breve{c}_{\left(k^{\prime} ; n^{\prime}\right)}^{*}\right]=r_{t}[\Delta k] r_{f}[\Delta n],
$$

where $\Delta k=k-k^{\prime}, \Delta n=n-n^{\prime}$, and $r_{t}[\Delta k]$ is the time-domain symbol-spaced autocorrelation function, which can be expressed as [10]

$$
r_{t}[\Delta k]=J_{0}\left(2 \pi f_{d m} \Delta k T_{B}\right),
$$

where $J_{0}(\cdot)$ is the zero-order Bessel function of the first kind and $f_{d m}$ is the maximum Doppler frequency. Furthermore, in (18) $r_{f}[\Delta n]$ is the frequency-domain symbol-spaced autocorrelation function, which can be expressed as [11]

$$
r_{f}[\Delta n]=\sum_{l=0}^{L-1} \sigma_{l}^{2} e^{-j 2 \pi \Delta n f_{0} \tau_{l}},
$$


where we have $f_{0}=1 /\left(N T_{b}\right)$ [12], $\tau_{l}$ represents the delay of the $l$ th multipath component and $\sigma_{l}^{2}$ is its average power. Accordingly, the autocorrelation function of $\tilde{c}_{(k ; n)}$ in (17) is given by

$$
\begin{aligned}
E\left[\tilde{c}_{(k ; n)} \tilde{c}_{\left(k^{\prime} ; n^{\prime}\right)}^{*}\right]= & r_{t}[\Delta k] r_{f}[\Delta n] \\
& +\frac{N N_{0}}{2} \frac{1}{\left|\breve{b}_{(k ; n)}\right|^{2}} \delta(\Delta k) \delta(\Delta n) .
\end{aligned}
$$

Furthermore, the cross-correlation between $\tilde{c}_{(k ; n)}$ and $\breve{c}_{(k ; n)}$ is given by

$$
E\left[\tilde{c}_{(k ; n)} \breve{c}_{\left(k^{\prime} ; n^{\prime}\right)}^{*}\right]=r_{t}[\Delta k] r_{f}[\Delta n]
$$

Based on (17) the channel estimation in the frequency-domain can be formulated as

$$
\hat{c}_{(k ; n)}=\mathbf{d}_{(k)}^{H} \tilde{\mathbf{c}}_{(k)} \quad 0 \leq n \leq N-1,
$$

where $\hat{c}_{(k ; n)}$ represents the estimate of $\breve{c}_{(k ; n)}$ in the frequency-domain and $d_{(k ; i)}$ represents the $i$ th filter coefficient. Furthermore, in (23) $\mathbf{d}_{(k)}=\left[d_{(k, 0)}, d_{(k ; 1)}, \cdots, d_{(k ; N-1)}\right]^{T}$ denotes the coefficient vector of the frequency-domain filter designed for estimating $\breve{c}_{(k ; n)}$ and $\tilde{\mathbf{c}}_{(k)}=\left[\tilde{c}_{(k ; 0)}, \tilde{c}_{(k ; 1)}, \cdots, \tilde{c}_{(k ; N-1)}\right]^{T}$ is the CTF vector having the elements given in (17). The coefficient vector $\mathbf{d}_{(k)_{o}}$ optimized in the MMSE sense can be obtained by using Wiener filtering according to [13], which may be expressed as

$$
\mathbf{d}_{(k)_{o}}=\mathbf{R}_{f}^{-1} \mathbf{r}_{f},
$$

where $\mathbf{R}_{f}$ is the $(N \times N)$-dimentional autocorrelation matrix of $\tilde{\mathbf{c}}_{(k)}$, which is given by

$$
\mathbf{R}_{f}=E\left[\tilde{\mathbf{c}}_{(k)} \tilde{\mathbf{c}}_{(k)}^{H}\right]
$$

while $\mathbf{r}_{f}$ is the $(N \times 1)$-dimentional cross-correlation vector given by

$$
\mathbf{r}_{f}=E\left[\tilde{\mathbf{c}}_{(k)} \breve{c}_{(k ; n)}^{*}\right]
$$

Furthermore, it can be shown that the minimum MSE $J_{f o(k ; n)}$ after frequency domain filtering can be expressed as

$$
J_{f o(k ; n)}=r_{f}[0]-\mathbf{r}_{f}^{H} \mathbf{R}_{f}^{-1} \mathbf{r}_{f} .
$$

After frequency-domain filtering in the MMSE sense, $\hat{c}_{(k ; n)}$ can be expressed as

$$
\hat{c}_{(k ; n)}=\breve{c}_{(k ; n)}+\zeta_{(k ; n)},
$$

where $\zeta_{(k ; n)}$ is a zero-mean process having a variance of $J_{f o(k ; n)}$, which represents the estimation error between $\hat{c}_{(k ; n)}$ and $\breve{c}_{(k ; n)}$. Furthermore, the autocorrelation function of $\hat{c}_{(k ; n)}$ at a given frequency can be expressed as

$$
E\left[\hat{c}_{(k ; n)} \hat{c}_{\left(k^{\prime} ; n\right)}^{*}\right]=r_{t}[\Delta k] r_{f}[0]+J_{f o(k ; n)} \delta(\Delta k)
$$

Note that when deriving (29) we exploited the property that $\zeta_{(k ; n)}$ is independent of $\hat{c}_{(k ; n)}$ and it is also indepent of $\zeta_{\left(k^{\prime} ; n\right)}$, when we have $k \neq k^{\prime}[10]$.

\section{B. Long Range Prediction of Wideband Channels}

Let us assume that a pilot symbol block having the same number of symbols as the data symbol block is inserted in every $M$ th tranmission block, i.e. the seperation between the pilot symbol blocks is $(M-1)$ transmission blocks. By long-range prediction, we mean that the CTF corresponding to the next pilot symbol block is predicted with the aid of all pilot blocks available from past, as illustrated in Fig. 1. Then, the specific CTF corresponding to the data symbol block to be demodulated is obtained by interpolating the CTF over $K$ number of consecutive pilot symbol blocks. Since the CTF $\breve{c}_{(k ; n)}$ experiences narrowband fading at a given frequency, after the FD channel estimation, a Kalman filter can be used to predict the future CTF at each frequency.

Specifically, for a fixed frequency component $n$, the CTF can be described by an AR model [14], which can be formulated as

$$
\breve{c}_{(k ; n)}=\sum_{m=1}^{p} a_{m} \breve{c}_{(k-m ; n)}+w_{(k ; n)},
$$

where $\left\{a_{m}\right\}$ represents the AR coefficients and $w_{(k ; n)}$ is the complex white Gaussian noise. Let us introduce the following notation:

$$
\begin{gathered}
\mathbf{F}_{(k-1 ; n)}=\left[\begin{array}{cccc}
a_{1} & \cdots & a_{p-1} & a_{p} \\
1 & \cdots & 0 & 0 \\
\vdots & \ddots & \vdots & \vdots \\
0 & \cdots & 1 & 0
\end{array}\right] ; \\
\breve{\mathbf{c}}_{(k ; n)}=\left[\breve{c}_{(k ; n)}, \breve{c}_{(k-1 ; n)}, \cdots, \breve{c}_{(k-p+1 ; n)}\right]^{T} ; \\
\mathbf{w}_{(k ; n)}=\left[w_{(k ; n)}, 0, \cdots, 0\right]^{T} .
\end{gathered}
$$

Then, the Kalman process equation can be described as [13]

$$
\breve{\mathbf{c}}_{(k ; n)}=\mathbf{F}_{(k-1 ; n)} \breve{\mathbf{c}}_{(k-1 ; n)}+\mathbf{w}_{(k ; n)},
$$

where $\mathbf{F}_{(k-1 ; n)}$ is the $(p \times p)$-dimentional transition matrix and $\mathbf{w}_{(k ; n)}$ is the $(p \times 1)$-dimentional process noise vector, respectively. According to (28) the Kalman measurement equation can be written as

$$
\hat{c}_{(k ; n)}=\mathbf{H}_{(k ; n)} \breve{\mathbf{c}}_{(k ; n)}+\zeta_{(k ; n)}
$$

where $\mathbf{H}_{(k ; n)}$ is a $(1 \times p)$-dimentional measurement matrix, which is given by

$$
\mathbf{H}_{(k ; n)}=[1,0, \cdots, 0] .
$$

Consequently, the Kalman filter based channel prediction can be formulated as [13]

$$
\grave{\mathbf{c}}_{[(k+1 ; n) \mid(k ; n)]}=\mathbf{F}_{(k ; n)} \grave{\mathbf{c}}_{[(k ; n) \mid(k-1 ; n)]}+\mathbf{G}_{(k ; n)} \boldsymbol{\alpha}_{(k ; n)},
$$

where $\grave{\mathbf{c}}_{[(k+1 ; n) \mid(k ; n)]}$ represents the MMSE prediction of $\breve{\mathbf{c}}_{(k+1 ; n)}$ based on all the $k$ observations of past pilot symbol blocks for a specific frequency component, while $\mathbf{G}_{(k ; n)}$ and $\boldsymbol{\alpha}_{(k ; n)}$ are the Kalman gain and the innovation process, respectively [13].

\section{Simulation Result}

In our simulations the average received $\mathrm{SNR}, \gamma_{b}$, evaluated before the DFT is defined as

$$
\gamma_{b}=\frac{\varepsilon}{N_{0}} \sum_{l=0}^{L-1} \sigma_{l}^{2},
$$




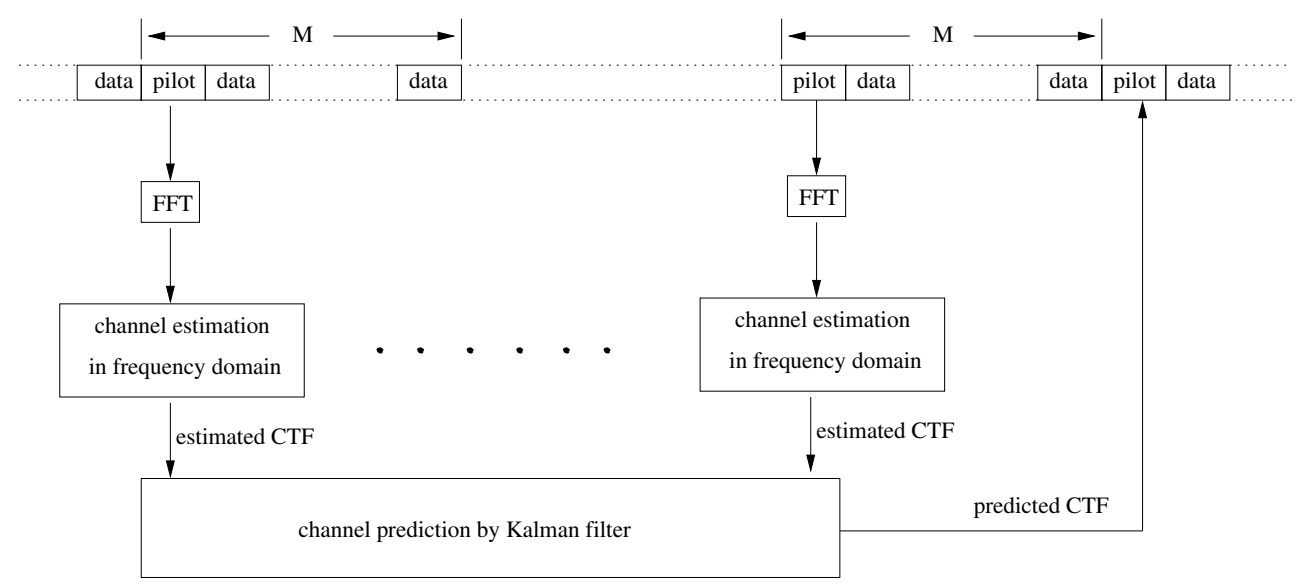

Fig. 1. Illustration of long range prediction for single-carrier systems.

where $\varepsilon$ is the average energy per symbol, $N_{0} / 2$ is the variance of the Gaussian noise and $\sigma_{l}^{2}=E\left[\left|c_{(k ; l)}\right|^{2}\right]$ is the average power of the $l$ th path. Furthermore, the pilot symbol block $\mathbf{b}_{k}$ is given by

$$
\mathbf{b}_{k}=[\underbrace{1, \cdots, 1}_{16}, \underbrace{-1,1, \cdots, 1}_{16}, \cdots, \underbrace{-1,1, \cdots, 1}_{16}, \underbrace{-1,1, \cdots, 1}_{16}] .
$$

Since the autocorrelation matrix may become singular when calculating the AR coefficients [14], a small positive value of $1 e-9$ is added to its main diagonal when calculating the AR model coefficients $\left\{a_{m}\right\}$ in our simulations.

In Fig.2, the CTF MMSE performance achieved after the frequencydomain CTF estimation according to (27) is demonstrated, when assuming that the maximum Doppler frequency was $f_{d m}=120 \mathrm{~Hz}$, the transmission block duration was $T_{B}=0.00025 \mathrm{~s}$, the number of multipath components was $L=10$, the length of the transmission block was $N=128$, the cyclic prefix was constituted by $u=9$ symbols, the power intensity was $\sigma_{l}^{2}=0.1$ for each multipath component, and the average SNR was $\gamma_{b}=20 d B$. we can see from Fig. 2 that the MMSE increases near-linearly with the frequency index $n$, when $n$ is lower than about 10. Furthermore, the MMSE remains near-constant, when the frequency index $n$ is in the range spanning from about 10 to 110 . Beyond this point, the MMSE linearly decreases with the frequency index $n$.

In Fig. 3 and Fig.4 the time-domain envelope of the true CTF $\breve{c}_{(k ; n)}$ and the Kalman filter based CTF prediction of $\grave{c}_{[(k ; n) \mid(k-1 ; n)]}$ are shown when assuming $\gamma_{b}=20 \mathrm{~dB}$ and $\gamma_{b}=30 \mathrm{~dB}$, respectively and assuming that the spacing of pilot symbol blocks was $M=5$, and the order of the Kalman filter was $p=20$. All other parameters are the same as in Fig.2. We can observe from Fig.3 and Fig.4 that when the SNR is sufficiently high, the Kalman filter based CTF predictor is capable of closely tracking the wideband channel frequency-domain's envelope, and the predicted CTF closely agrees with the true CTF.

Finally, in Fig.5 we evaluated the achievable CTF MMSE performance versus the time expressed in terms of the number of the pilot symbol blocks used by for the Kalman filter based CTF predictor. We assumed that the SNR was $\gamma_{b}=20 d B$ and $\gamma_{b}=30 d B$, respectively. All other parameters are the same as in Fig. 3 and Fig. 4. In Fig. 5 the CTF MMSE corresponding to the 0th and 50th frequency components were recorded. We can observe from the results of Fig. 5 that the MMSE reaches a certain residual value, when the number of pilot symbol blocks increases. Moreover, for a fixed SNR value of $\gamma_{b}$, the

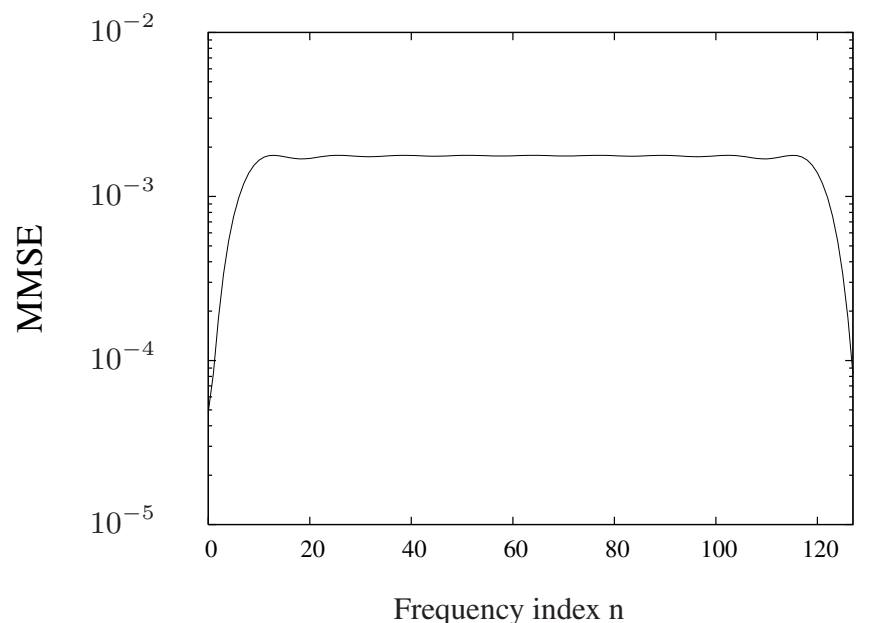

Fig. 2. The CTF MMSE achieved after frequency-domain CTF estimation, when assuming that the maximum Doppler frequency was $f_{d m}=120 \mathrm{~Hz}$, the transmission block duration was $T_{B}=0.00025 \mathrm{~s}$, the number of multipath components was $L=10$, the length of the transmission block was $N=128$, the length of the cyclic prefix was $u=9$, the power intensity was $\sigma_{l}^{2}=0.1$ for each multipath component and the average SNR was $\gamma_{b}=20 d B$.

MMSE corresponding to the 0th frequency component is lower than that corresponding to the 50th frequency component. The reason for the above observation is that a different MMSE was obtained for different frequency components after the frequency domain estimation, as shown in Fig.2.

\section{REFERENCES}

[1] L. Hanzo, C. H. Wong and M. S. Yee "Adaptive wireless transceivers: turbo-coded, turbo-equalized and space-time coded TDMA, CDMA, and OFDM Systems," John Wiley \& Sons - IEEE Press, 2002.

[2] L. Hanzo, M. Münster and B. J. Choi and T. Keller "OFDM and MCCDMA for broadband multi-user communications, WLANs and broadcasting," John Wiley \& Sons - IEEE Press, 2003.

[3] A. Duel-Hallen, S. Hu and H. Hallen, "Long range prediction of fading signals: enabling adaptive transmission for mobile radio channels," IEEE Signal Processing Magzine, vol. 17, pp. 62-75, May 2000.

[4] L. Dong, G. Xu and H. Ling, "Prediction of fast fading mobile radio channels in wideband communication systems," Global Telecommunications Conference, pp. 3287 - 3291, November 2001. 


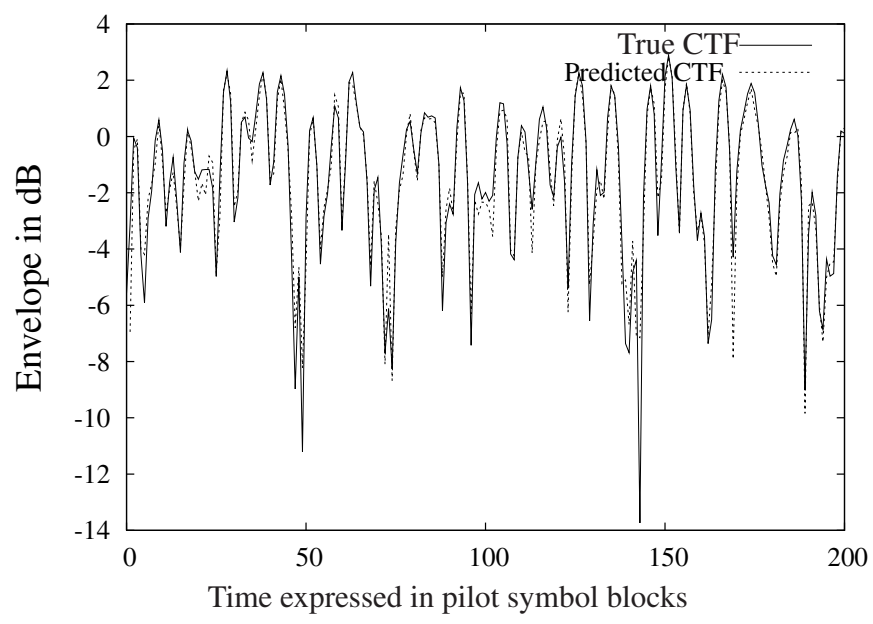

Fig. 3. The true CTF magnitude and the Kalman filter based predicted CTF of the 50th frequency component after frequency-domain estimation, when assuming that the spacing of pilot symbol blocks was $M=5$ transmission blocks, the maximum Doppper frequency was $f_{d m}=120 \mathrm{~Hz}$, the transmission block duration was $T_{B}=0.00025 \mathrm{~s}$, the number of multipath components was $L=10$, the length of the transmission block was $N=128$, the length of the cyclic prefix was $u=9$, the power intensity was $\sigma_{l}^{2}=0.1$ for each multipath component and the SNR was $\gamma_{b}=20 d B$. Furthermore, the order of the Kalman filter was $p=20$.

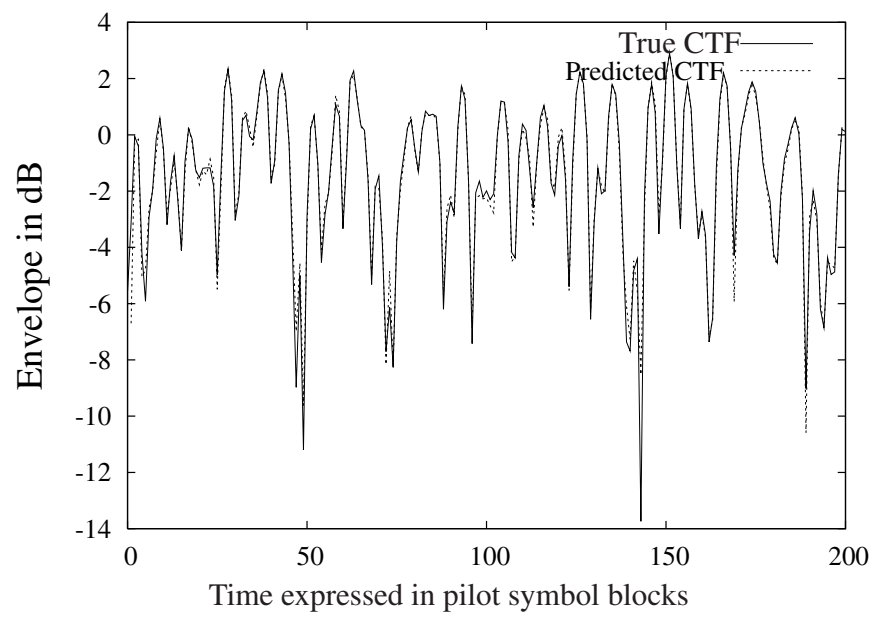

Fig. 4. The true CTF envelope and the Kalman filter based predicted CTF of the 50th frequency component after frequency-domain estimation, when assuming that the spacing of pilot symbol blocks was $M=5$ transmission blocks, the maximum Doppper frequency was $f_{d m}=120 \mathrm{~Hz}$, the transmission block duration was $T_{B}=0.00025 \mathrm{~s}$, the number of multipath components was $L=10$, the length of the transmission block was $N=128$, the length of the cyclic prefix was $u=9$, the power intensity was $\sigma_{l}^{2}=0.1$ for each multipath component and the SNR was $\gamma_{b}=30 d B$. Furthermore, the order of the Kalman filter was $p=20$.

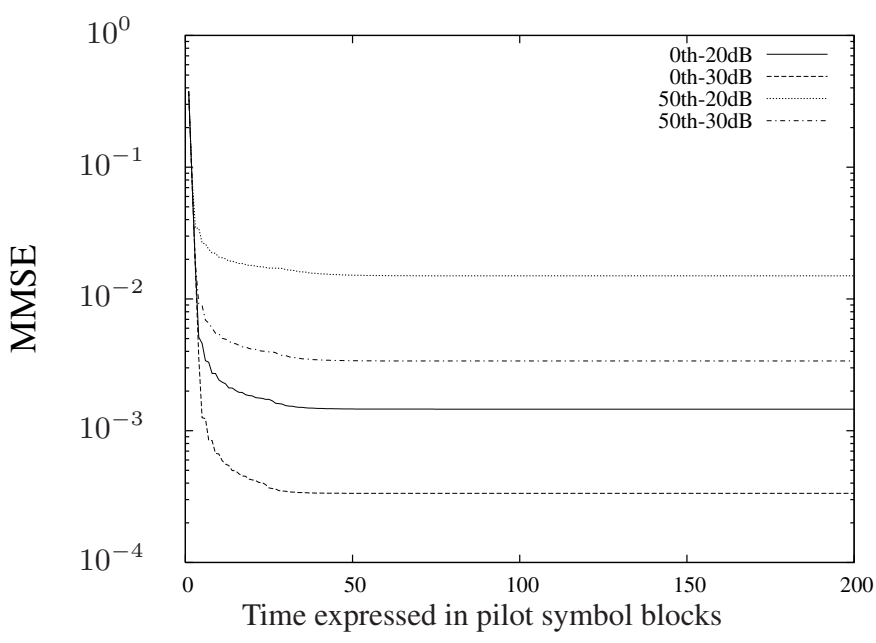

Fig. 5. The CTF MMSE versus the time expressed in terms of the number of the pilot symbol blocks for the Kalman filter based long-range CTF prediction after the frequency domain estimation of the 0th frequency component and 50 th frequency component, when assuming that the spacing of pilot symbol block was $M=5$, the maximum Doppler frequency was $f_{d m}=120 \mathrm{~Hz}$, the transmission block duration was $T_{B}=0.00025 \mathrm{~s}$, the number of multipath components was $L=10$, the length of transmission block was $N=128$, the length of the cyclic prefix was $u=9$, the power intensity was $\sigma_{l}^{2}=0.1$ for each multipath component, the SNR before employing the DFT to transmission block was $\gamma_{b}=20 d B$ and $\gamma_{b}=30 d B$, respectively. Furthermore, the order of the Kalman filter was $p=20$.

[5] S. Semmelrodt and R. Kattenbach, "Application of spectral estimation techniques to 2-D fading forecast of time-variant channels," COST 273 $T D(01) 034$, October 2001.

[6] S. Semmelrodt and R. Kattenbach, "A 2-D fading forecast of time-variant channels based on parametric modelling techniques," The 13th IEEE International Symposium on Personal, Indoor and Mobile Radio Communications, pp. 1640 - 1644, September 2002.

[7] T. Hwang and Y. Li, "Iterative cyclic prefix reconstruction for coded single-carrier systems with frequency-domain equalization (SC-FDE)," IEEE Vehicular Technology Conference 2003-Spring, pp. 1841 - 1845, April 2003.

[8] N. Al-Dhahir, "Single-carrier frequency-domain equalization for spacetime block-coded transmissions over frequency-selective fading channels," IEEE Communications Letters, vol. 5, pp. 304 - 306, July 2001.

[9] D. Falconer, S. L. Ariyavisitakul, A. Benyamin-Seeyar and B. Eidson, "Frequency domain equalization for single-carrier broadband wireless systems," IEEE Communications Magazine, vol. 40, pp. 58 - 66, April 2002.

[10] P. Hoeher, S. Kaiser and P. Robertson, "Pilot-symbol-aided channel estimation in time and frequency," IEEE Global Telecommunications Conference, pp. 90-96, November 1997.

[11] W. G. Jeon, K. H. Paik and y. S. Cho, "Two-dimensional MMSE channel estimation for OFDM systems with transmitter diversity," IEEE Vehicular Technology Conference 2001-Fall, pp. 1682 - 1685, October 2001.

[12] H. Witschnig, T. mayer, A. Springer, L. Maurer, M. Huemer and R. Weigel, "The advantages of a known sequence versus cyclic prefix in a SC/FDE system," The 5th International Symposium on Wireless Personal Multimedia Communications, pp. 1328 - 1332, October 2002.

[13] S. Haykin, Adaptive Filter Theory. Prentice Hall, Inc, 2002.

[14] K. E. Baddour and N. C. Beaulieu, "Autoregressive models for fading channel simulation," IEEE Global Telecommunications Conference, pp. 1187 - 1192, November 2001. 